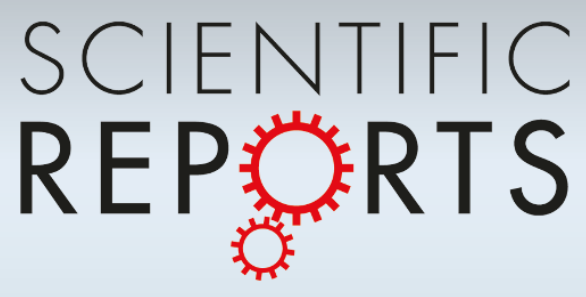

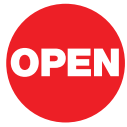

SUBJECT AREAS: PHYSICS

NONLINEAR OPTICS

OPTICAL PHYSICS

FIBRE OPTICS AND OPTICAL COMMUNICATIONS

Received

29 March 2012

Accepted

25 May 2012

Published

18 June 2012

Correspondence and requests for materials should be addressed to J.M.D. (john.dudley@ univ-fcomte.fr)

\section{Observation of Kuznetsov-Ma soliton dynamics in optical fibre}

\author{
B. Kibler' , J. Fatome' , C. Finot ', G. Millot ', G. Genty ${ }^{2}$, B. Wetzel ${ }^{3}$, N. Akhmediev ${ }^{4}$, F. Dias ${ }^{5}$ \& J. M. Dudley ${ }^{3}$
}

'Laboratoire Interdisciplinaire Carnot de Bourgogne, UMR 6303 CNRS - Université de Bourgogne, Dijon, France, ${ }^{2}$ Tampere University of Technology, Optics Laboratory, Fl-33101 Tampere, Finland, ${ }^{3}$ Institut FEMTO-ST, UMR 6174 CNRS- Université de Franche-Comté, 25030 Besançon, ${ }^{4}$ Optical Sciences Group, Research School of Physics and Engineering, Institute of Advanced Studies, The Australian National University, Canberra ACT 0200, Australia, ${ }^{5}$ School of Mathematical Sciences, University College Dublin, Belfield, Dublin 4, Ireland.

The nonlinear Schrödinger equation (NLSE) is a central model of nonlinear science, applying to hydrodynamics, plasma physics, molecular biology and optics. The NLSE admits only few elementary analytic solutions, but one in particular describing a localized soliton on a finite background is of intense current interest in the context of understanding the physics of extreme waves. However, although the first solution of this type was the Kuznetzov-Ma (KM) soliton derived in 1977, there have in fact been no quantitative experiments confirming its validity. We report here novel experiments in optical fibre that confirm the KM soliton theory, completing an important series of experiments that have now observed a complete family of soliton on background solutions to the NLSE. Our results also show that KM dynamics appear more universally than for the specific conditions originally considered, and can be interpreted as an analytic description of Fermi-Pasta-Ulam recurrence in NLSE propagation.

1 he nonlinear Schrödinger equation (NLSE) provides a central description of a variety of nonlinear localization effects, and has been intensively studied in various contexts in nonlinear science ${ }^{1,2}$. Since the pioneering analysis of Zakharov and Shabat in 1971, there has been wide and continued investigation into the properties of analytic soliton solutions of the $\mathrm{NLSE}^{3}$, both for their intrinsic scientific interest, as well as for their potential to provide new insights into important applications such as optical propagation in nonlinear waveguides ${ }^{4}$. For the case of a self-focussing nonlinearity, the most celebrated solution of this type is very likely the propagation-invariant hyperbolic secant bright soliton, but there is also an extensive literature studying various types of solitons on finite background (SFB) consisting of a localized nonlinear structure evolving upon a nonzero background plane wave ${ }^{5-12}$.

Solitons on finite background have recently attracted significant interest as their localization dynamics have been proposed as an important mechanism underlying the formation of the infamous extreme amplitude freak waves on the surface of the ocean ${ }^{13-16}$. Much of this work has also been motivated by a parallel research effort using nonlinear optical fibre systems to implement controlled experiments studying NLSE dynamics and rogue waves in a purely optical context ${ }^{17}$. Many of these recent studies have focussed on the characteristics of the Akhmediev breather, a particular SFB solution which is excited from a weak periodic modulation and which is localized in the longitudinal dimension as it undergoes growth and decay ${ }^{9}$. Experiments in optics have demonstrated important links to modulation instability ${ }^{18}$ and Fermi Pasta Ulam recurrence ${ }^{19}$, the nonlinear evolution process whose study essentially founded the field of computational nonlinear science ${ }^{20}$. Another significant application of the theory of Akhmediev breathers has been to design experiments generating the rational Peregrine soliton, an important and limiting case of a SFB solution that is localised in both transverse and longitudinal dimensions ${ }^{8,21,22}$.

The first SFB solution to the NLSE that was obtained is now known as the Kuznetsov-Ma (KM) soliton which, in contrast to the Akhmediev breather, undergoes periodic evolution with propagation. Surprisingly, however, although this solution has been known since $1977^{5-7}$, the theoretical predictions describing KM soliton dynamics have in fact never been the subject of detailed experimental study. Certainly experiments on periodic wave train dynamics have been reported since the early years of studies in nonlinear hydrodynamics, but the results presented have been purely qualitative or have been compared only with numerical studies of the NLSE ${ }^{23-26}$. To our knowledge, there have been no explicit comparisons between experiments studying periodic nonlinear NLSE wave evolution and the theory of the KM soliton on finite background.

In this paper, we address this issue directly, and present the first experimental confirmation of the pioneering theoretical studies of the KM soliton solution of the NLSE. In what follows, we first review the properties of the 
(a) Akhmediev breather $a=0.25$

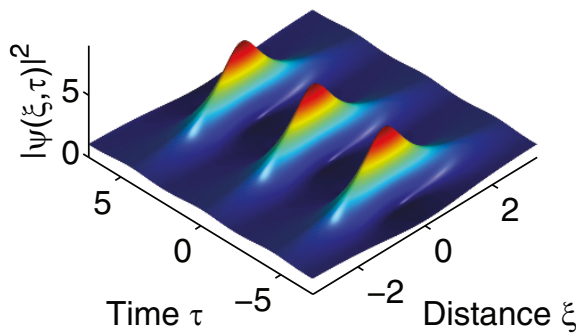

(b) Peregrine soliton

$a=0.5$

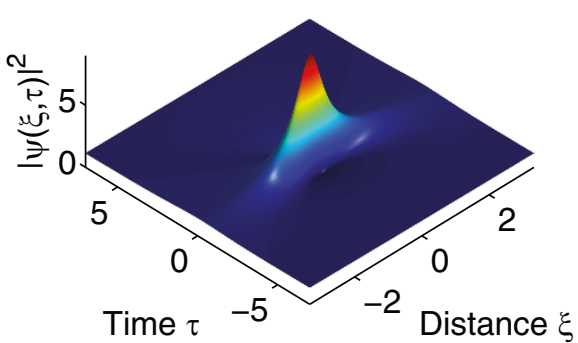

(c) Kuznetsov-Ma soliton

$a=1$

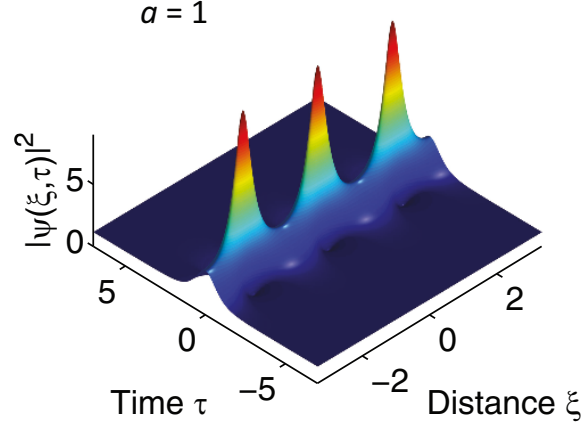

Figure 1 Analytic solutions of the NLSE from Eq. (2) with different values of parameter $\boldsymbol{a}$ as indicated illustrating the three different classes of primary soliton on finite background solutions of the NLSE.

different SFB solutions to the NLSE under ideal conditions, and then use numerical simulations to show how the dynamics of the KM soliton also appear in the longitudinal growth and decay of the individual modulation cycles of a strongly modulated initial plane wave in optical fibre. These simulations allow us to show how KM dynamics appear under conditions much wider than those originally considered, allowing us to design experiments using tailored initial conditions of a modulated optical source injected in optical fibre to confirm the KM soliton theory. Specifically, using ultrafast metrology and fibre cutback experiments, we measure the evolving temporal profile with propagation distance along the fibre, allowing us to perform a direct comparison between the generated temporal profile and its longitudinal evolution, and the analytic prediction of the KM solution. These results now complete an important series of experiments that have now observed a complete family of soliton on background solutions to the NLSE, and lead to the interpretation of the KM soliton as an analytic limit of the Fermi-Pasta-Ulam recurrence phenomenon in nonlinear fibre propagation.

\section{Results}

The Akhmediev, Peregrine and Kuznetsov-Ma solutions compared. We first review the different SFB solutions to the NLSE which have motivated our experimental studies. The dimensionless self-focussing NLSE is written:

$$
i \frac{\partial \psi}{\partial \xi}+\frac{1}{2} \frac{\partial^{2} \psi}{\partial \tau^{2}}+|\psi|^{2} \psi=0
$$

where $\psi(\xi, \tau)$ is a wave group or pulse envelope which is a function of $\xi$ (a propagation distance or longitudinal variable) and $\tau$ (a co-moving time or transverse variable). Transformation to experimental parameters is given in Section $C$ below. Using the notation of Ref. ${ }^{9}$, a general SFB solution of the NLSE can be written compactly as follows:

$$
\psi(\xi, \tau)=e^{i \xi}\left[1+\frac{2(1-2 a) \cosh (b \xi)+i b \sinh (b \xi)}{\sqrt{2 a} \cos (\omega \tau)-\cosh (b \xi)}\right]
$$

Here, the single governing parameter $a$ determines the physical behaviour of the solution through the function arguments $b=[8 a$ $(1-2 a)]^{1 / 2}$ and $\omega=2(1-2 a)^{1 / 2}$.

We plot the solution of Eq. (2) for different values of $a$ in Fig. 1. For $a<1 / 2$ as shown in Fig. 1(a) the solution describes the Akhmediev breather, and $\omega$ and $b$ are real with physical significance as a modulation frequency and exponential growth and decay rate. We see clearly the growth and decay cycle of the initial weak periodic modulation. For $a=1 / 2$ as in Fig. 1(b), the solution describes the Peregrine soliton corresponding to the low frequency limit of the Akhmediev breather which in this case is localized in both transverse and longitudinal dimensions. For $\mathrm{a}>1 / 2$ as in Fig. 1(c), the solution describes the KM soliton where the parameters $\omega$ and $b$ become imaginary such that the hyperbolic trigonometric functions in Eq. (2) become ordinary circular functions and vice-versa. It is this that leads to the contrasting localization and periodicity characteristics and different physics of the Akhmediev and KM solutions.

Observing KM dynamics: Modulation Instability with strong modulation amplitude. Exciting an ideal Akhmediev breather requires a specific choice of weakly modulated continuous wave as initial condition ${ }^{27}$, and deviation from ideal initial conditions (e.g. with a large amplitude initial modulation) yields more complex behaviour. Although general solutions for complex evolution dynamics can be found using Jacobi elliptic functions ${ }^{10}$, we have found that the longitudinal dynamics observed with a large initial modulation can in fact be described and interpreted in terms of the expected longitudinal evolution of a KM soliton. It is this realization that has allowed us to design experiments to excite KM soliton dynamics under controlled conditions for the first time.

This approach is shown in Fig. 2, where we use numerical integration of the NLSE to compare the evolution of an input field based on an exact KM soliton with that of a suitably designed stronglymodulated field that approximates the KM soliton over each modulation cycle (see Methods). We first compare the two initial profiles (a) Input field construction

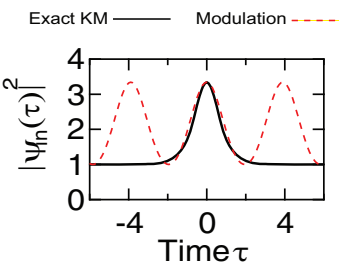

(b) Simulation for Modulated Input

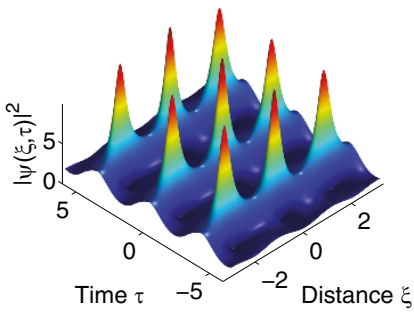

(c) Detail over Central Region
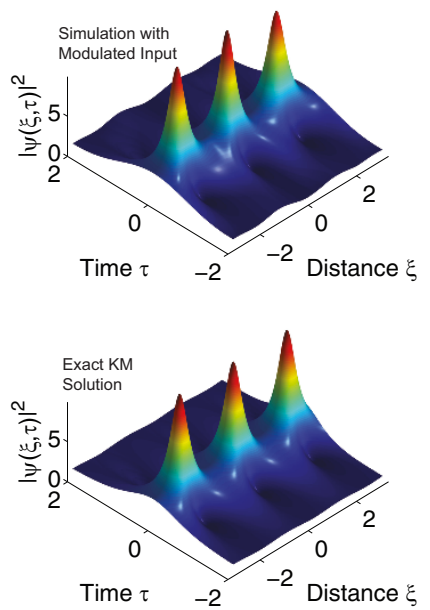

Figure $2 \mid$ (a) Input field comparing ideal KM solution and modulation approximation. (b) Integrating the NLSE for the modulated input field shows complex evolution. (c) The evolution for the central region of the NLSE simulation results agrees very well with the evolution of the ideal KM soliton. 
used in the simulations in Fig. 2(a). Specifically, we choose the initial conditions of a strongly-modulated continuous wave (dashed line) such that the central modulation cycle overlaps with the KM soliton pulse above the background as shown (solid line) at a point of minimal intensity in its evolution. The governing parameter of the KM soliton in this case is $a_{K M}=1$.

In Fig. 2(b) we show results from the numerical integration of the NLSE for the strongly modulated input field described above where we see complex and biperiodic evolution. Significantly, however, when we compare a detailed view of the evolution along $\xi$ of the central cycle of the modulated signal with the corresponding exact evolution for the KM soliton as we show in Fig. 2(c), we see near identical periodic evolution in the two cases. (Of course, the choice of the central modulation cycle here is arbitrary; all cycles of the modulated field evolve in the same way along $\xi$ ). This now allows us to interpret the periodic longitudinal evolution generated from an initial strong modulation in terms of the evolution of an equivalent KM soliton. Identifying this physical equivalence is extremely important, as it shows the universality of KM dynamics in NLSE propagation, even under conditions very different from those originally considered. Moreover, since such periodic evolution in the NLSE is wellknown to be an example of Fermi-Pasta-Ulam recurrence ${ }^{19}$, we can also now interpret the KM soliton result as an analytic description of this process.

Experimental Setup and Results. From an experimental viewpoint, the results above have allowed us to design experiments where the predictions of the analytic KM soliton theory can be tested for the first time. Our experimental set up is shown in Fig. 3 (see Methods). We use high speed telecommunications-grade components to strongly modulate a continuous wave $1550 \mathrm{~nm}$ laser diode. The modulation strength and period are chosen such that the characteristics of each cycle match a particular KM soliton as shown in Fig. 2(a). We aim to synthesize an input field at the fibre input $z=0$ corresponding to a KM soliton of governing parameter $a_{K M}$. The initial conditions are written in dimensional form as $A_{\text {mod }}(z=0, T)=\sqrt{P_{0}}+\sqrt{P_{0}}\left(\sqrt{2 a_{K M}}-1\right)\left(1+\cos \left(\Omega_{\bmod } T\right)\right) \quad$ such that the field varies between minimum "background" amplitude of

(a) Experimental Setup

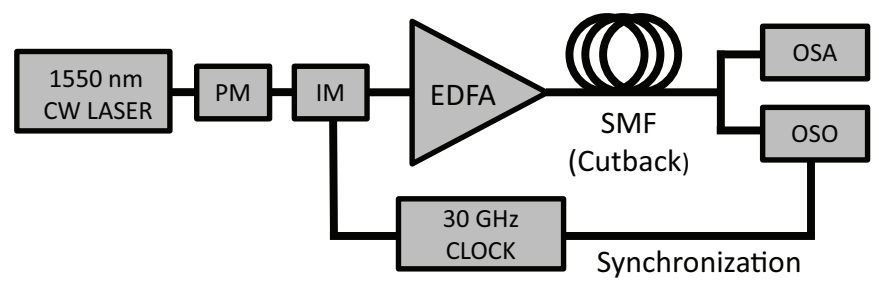

(b) Initial Conditions

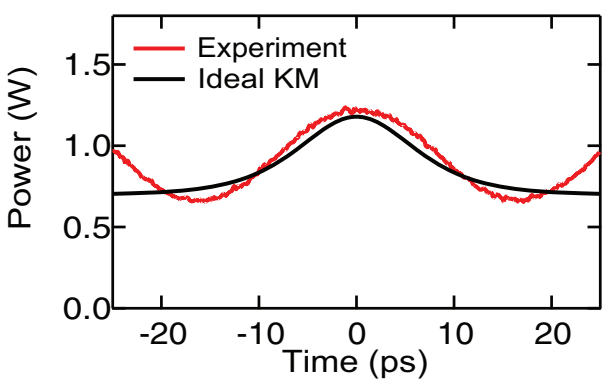

Figure $3 \mid$ (a) Experimental setup. PM: phase modulator. IM: intensity modulator. EDFA: Erbium doped fibre amplifier. SMF: single mode fibre: OSA: optical spectrum analayser. OSO: optical sampling oscilloscope. (b) Ideal KM soliton at minimum intensity for $a_{K M}=0.66$ (black) compared with the experimentally synthesized modulated field (red).
$\sqrt{P_{0}}$ and a maximum value of $\sqrt{P_{0}}+2 \sqrt{P_{0}}\left(2 a_{K M}-1\right)$. Note that $P_{0}$ here is the background field power in $\mathrm{W}$. We study propagation in SMF-28 optical fibre with group velocity dispersion $\beta_{2}\left[\mathrm{~s}^{2} \mathrm{~m}^{-1}\right]$ and nonlinearity $\gamma\left[\mathrm{W}^{-1} \mathrm{~m}^{-1}\right]$. The dimensional field $A(z, T)\left[\mathrm{W}^{1 / 2}\right]$ is: $A=\sqrt{P_{0}} \psi$ and defining a timescale $T_{0}=\left(\left|\beta_{2}\right| L_{\mathrm{NL}}\right)^{1 / 2}$, the dimensional distance time $T[\mathrm{~s}]$ is $T=\tau T_{0}$. We also define a characteristic length $L_{\mathrm{NL}}=\left(\gamma P_{0}\right)^{-1}$ such that the dimensional distance $z[\mathrm{~m}]$ is $z=\xi L_{N L}+z_{p} / 2$ (where $z_{p}$ corresponds to one period of the KM cycle so that our initial conditions above are injected at $z=0$.)

In our experiments, we characterized the field propagation by cutting the fiber back in steps of $\sim 200 \mathrm{~m}$ from its original length. This allows quantitative measurements of evolution with distance that we can compare with the theoretically-expected behaviour of an ideal KM soliton. The evolving field profile was characterized for each fibre length using an ultrafast optical sampling oscilloscope (OSO) and a high dynamic range optical spectrum analyser (OSA). A phase modulator (PM) was used to mitigate the effects of Brillouin scattering, and an erbium-doped fibre amplifier (EDFA) was used to obtain average powers up to $1 \mathrm{~W}$. The modulation strength (at $\sim 30.5 \mathrm{GHz}$ ) was optimized to yield individual modulation cycles that provided a very good fit to an ideal KM of $0.7 \mathrm{~W}$ background and $1.2 \mathrm{~W}$ peak power with the target $\mathrm{KM}$ soliton parameter here $a_{K M}=0.66$. The modulated input field synthesized experimentally and the ideal KM soliton are compared in Fig. 3(b). The SMF-28 fibre length used corresponded to one period of the expected KM cycle $z_{p}=5.3 \mathrm{~km}$. Higher values of $a_{K M}$ and longer fibre lengths could not be used in our experiments because of limitations due to fibre loss and Brillouin scattering.

Our experimental results are shown in Fig. 4. Figure 4(a) is a false color plot of the measured temporal intensity profiles at each propagation distance, compared with the profiles of an ideal KM soliton. There is clearly very good qualitative agreement, and this is confirmed quantitatively in Fig. 4(b) by comparing the instantaneous power at the centre of the modulation cycle $(T=0)$ from experiment (red circles) with the corresponding power evolution for an ideal KM soliton (solid line). Note that there are no free parameters used when plotting the expected theoretical KM soliton evolution. The small discrepancy between experiment and theory arises from fibre loss, but performing numerical simulations of the propagation of the experimental input field including loss (blue dashed line) reproduces experiment near-exactly.

Finally, in Fig. 4(c) we plot the explicit comparison between experimental and ideal KM soliton profiles in both the time and frequency domains at the point of maximum temporal compression when $z=z_{p} / 2$. The agreement between experiment (red), theory (black), and numerical modeling (blue) for both temporal intensity and spectrum is again very good, and the experimental characterization shows both the localized soliton and the background components of the KM soliton at this point. Note that the theoretical spectrum (see Methods) plotted corresponds only to the time-varying envelope component of the KM soliton and that the delta-function component at the pump wavelength (from the infinite background of the ideal solution) is not shown. Nonetheless, there is remarkable agreement between the measured decay of the spectral sidebands of the experimentally observed KM soliton and theory.

\section{Discussion}

The results in Fig. 4 show excellent agreement between the nonlinear longitudinal evolution of a modulation cycle of a strongly modulated continuous wave and the dynamics of an ideal KM soliton. The fact that the KM soliton theory describes the evolution of individual modulation cycles is an essential conclusion of this paper and indeed, the identical evolution observed for each cycle of the evolving field leads us to conclude that these tailored initial conditions actually 
(a) Evolution of Central Lobe

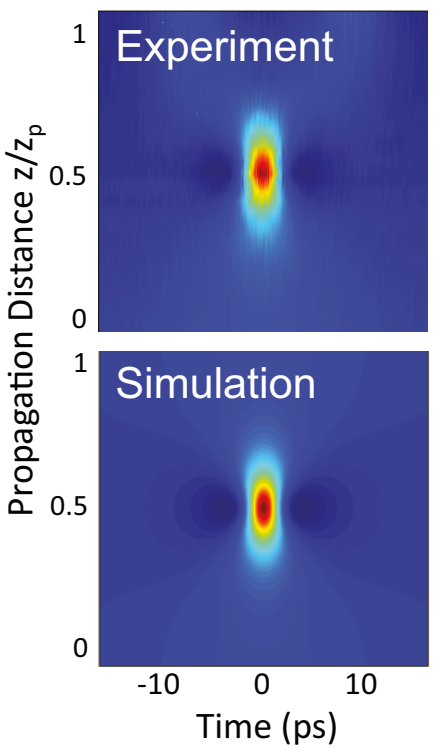

(b) Power evolution with distance

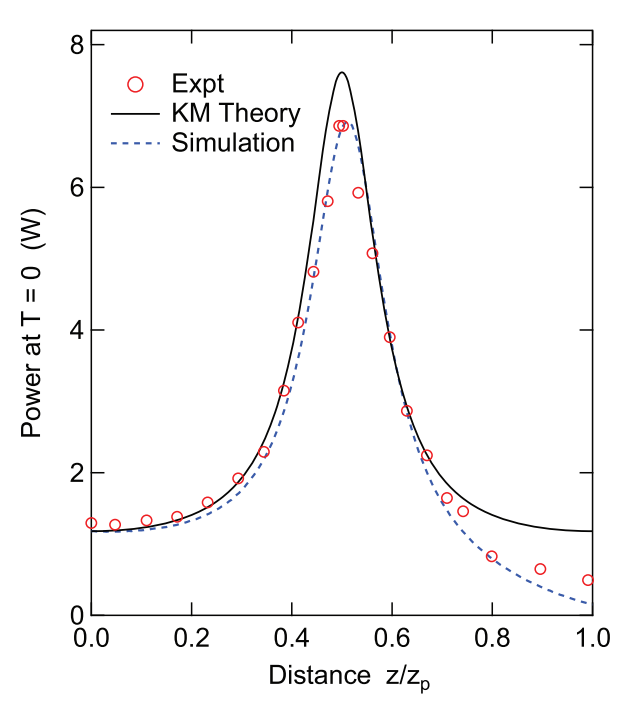

(c) Experiment and Theory at $z / z_{p}=0.5$
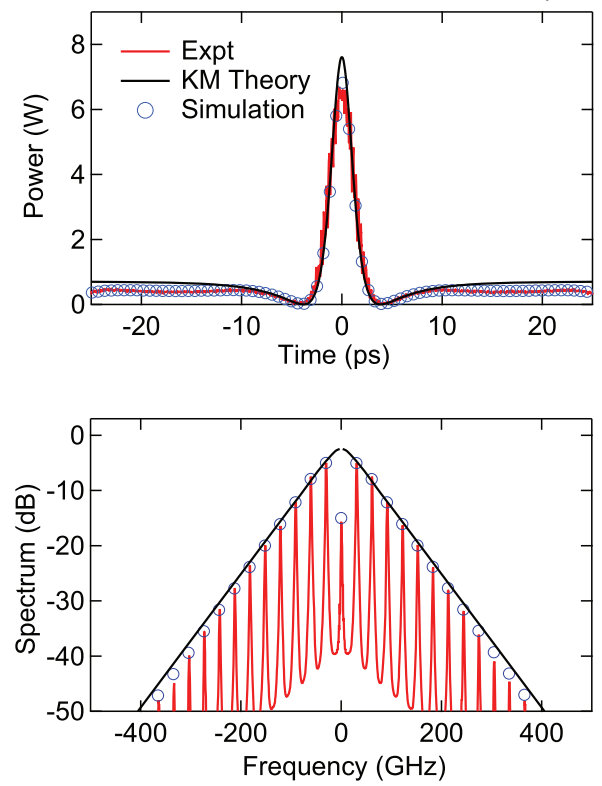

Figure $4 \mid$ (a) False color plot of experimental and theoretical intensity evolution with propagation distance. (b) plots the evolution of the power at the centre of the modulation cycle as a function of normalised distance $\left(z_{p}=5.3 \mathrm{~km}\right)$ comparing experiment (red), the theoretical evolution of the KM soliton (black) and simulation (blue). (c) compares time-domain and frequency-domain properties of the KM soliton for maximum temporal compression at $z=z_{p} / 2$.

yield a high repetition rate train of Kuznetsov-Ma solitons with high quality compressed pulse characteristics.

These results complete a series of recent studies in optics that have now observed all three of the primary class of soliton on finite background solutions to the NLSE: the Akhmediev breather, the Peregrine soliton - and here - the Kuznetsov Ma soliton. In addition, the fact that we show that KM dynamics appear more universally than for the specific conditions considered in the original theory is further support of recent generalisations of the soliton concept beyond those based on strict mathematical considerations ${ }^{28}$. Our results also establish a further link between the dynamics of solitons on finite background and the seminal Fermi-Pasta-Ulam recurrence phenomenon of nonlinear dynamics. In fact, our results support an important interpretation of the KM soliton as a special case of analytic descriptions of NLSE evolution which provide a general framework to describe the Fermi-Pasta-Ulam process in NLSE systems ${ }^{1,10}$.

In conclusion, it is interesting to remark that whilst a 35 year interval between original theoretical studies and experimental confirmation may seem surprising, it is only recently that the importance of these soliton on background solutions has been widely appreciated within physics. Within the nonlinear optics community in particular, it is worth noting that high quality pulse characterisation in optics is a relatively recent development ${ }^{29-31}$, and an important implication of our work is to anticipate that experiments using optical systems will continue to provide an important and highly convenient means of testing fundamental theories of nonlinear wave dynamics.

\section{Methods}

To describe the modelling of a KM soliton by a periodic modulation over a single cycle, it is useful to write Eq. (3) for $a>1 / 2$ in terms of real arguments $B$ and $\Delta \tau$ where $b=i \sqrt{8 a(2 a-1)}=i B$ and $\omega=i 2 \sqrt{(2 a-1)}=i \Delta \tau^{-1}$

$$
\psi(\xi, \tau)=e^{i \xi}\left[1+\frac{2(1-2 a) \cos (B \xi)+i B \sin (B \xi)}{\sqrt{2 a} \cosh (\tau / \Delta \tau)-\cos (B \xi)}\right]
$$

The KM solution evolves periodically with period $\Delta \xi=2 \pi / B=2 \pi / \sqrt{8 a(2 a-1)}$. Transverse localisation is determined by $\Delta \tau$. Ignoring a phase offset, the minimum amplitude solution is:

$$
\psi_{\min }(\tau)=1+\frac{2(2 a-1)}{\sqrt{2 a} \cosh (\tau / \Delta \tau)+1}
$$

and the solid line in Fig. 2(a) plots $\left|\psi_{\min }(\tau)\right|^{2}$ for $a=1$. The full width at half maximum $\left(\Delta T_{\text {FWHM }}\right)$ of the time-varying amplitude above background of Eq. (4) is used to determine numerically the optimal frequency for a modulated field approximation: $\Omega_{\text {mod }}=2 \pi /\left(2.22 \Delta T_{\text {FWHM }}\right)$. The compressed pulse at the point of maximum compression is:

$$
\psi_{\max }(\tau)=1+\frac{2(1-2 a)}{\sqrt{2 a} \cosh (\tau / \Delta \tau)-1}
$$

with Fourier transform used to calculate the continuous spectrum in Fig 4 (c) analytically given by: $\tilde{\Psi}(v)=\delta(0)-2 \pi \sinh \left(2 \pi \Delta \tau \cos ^{-1}[-1 / \sqrt{2 a}] v\right) / \sinh \left(2 \pi^{2} \Delta \tau v\right)$ where $v$ is the reciprocal Fourier variable of $\tau$.

Experiments used a tunable high repetition rate optical source (Photline Technologies ICB SoFast ModBox), including a laser diode at $1554.9 \mathrm{~nm}$ modulated at $\sim 30.5 \mathrm{GHz}$ by means of an Intensity Modulator (with significant energy in only two sidebands about the pump), a $30 \mathrm{dBm}$ erbium-doped fiber amplifier and a phase modulator which broadens the laser linewidth to $\sim 43 \mathrm{MHz}$ to suppress Brillouin scattering. Spectral measurements (Yokogawa - AQ6370 OSA) were carried out with $0.02 \mathrm{~nm}$ resolution bandwidth and the optical sampling oscilloscope (Picosolve PSO-101) had 0.8 ps resolution. The SMF-28 fibre had parameters: $\beta_{2}=-21.8 \mathrm{ps}^{2} \mathrm{~km}^{-1}$,

$\beta_{3}=0.012 \mathrm{ps}^{3} \mathrm{~km}^{-1}, \gamma=1.3 \mathrm{~W}^{-1} \mathrm{~km}^{-1}$ and $0.2 \mathrm{~dB} / \mathrm{km}$ loss. With $P_{0}=0.7 \mathrm{~W}$, the normalization constants $L_{\mathrm{NL}}=1.1 \mathrm{~km}$ and $T_{0}=4.9 \mathrm{ps}$. The KM periodicity is $L_{N L} \Delta \xi$ $=5.3 \mathrm{~km}$.

Numerical integration results in Fig 2 were based on a standard split-step scheme. Accurate simulation of the modulated input field required discretization so that modulation harmonics fall exactly on frequency-domain grid points. The numerical simulations in Fig. 4 also included loss, third-order dispersion and a $-50 \mathrm{~dB}$ noise background to model the effect of the EDFA, but the loss was found to be the primary reason for deviation from theory.

1. Akhmediev, N. \& Ankiewicz, A. Solitons, Nonlinear Pulses and Beams. Chapman and Hall, London (1997).

2. Dauxois, Th. \& Peyrard, M. Physics of Solitons (Cambridge University Press, Cambridge, 2006).

3. Zakharov, V. E. \& Shabat, A. B. Exact theory of two-dimensional self-focusing and one-dimensional self-modulation of waves in nonlinear media. Zh. Eksp. Teor. Fiz. 61, 118-134 (1971).

4. Agrawal, G. P. Nonlinear Fiber Optics (4th Edition, Academic Press, Boston, 2007).

5. Kuznetsov, E. Solitons in a parametrically unstable plasma. Sov. Phys. Dokl. 22, 507-508 (1977). 
6. Kawata, T. \& Inoue, H. Inverse scattering method for the nonlinear evolution equations under nonvanishing conditions. J. Phys. Soc. Japan 44, 1722-1729 (1978).

7. Ma, Y. C. The perturbed plane-wave solutions of the cubic Schrödinger equation. Stud. Appl. Math. 60, 43-58 (1979).

8. Peregrine, D. H. Water waves, nonlinear Schrödinger equations and their solutions. J. Austral. Math. Soc. Ser. B 25, 16-43 (1983).

9. Akhmediev, N. \& Korneev, V. I. Modulation instability and periodic solutions of the nonlinear schrödinger equation. Theor. Math. Phys. 69, 1089-1093 (1986).

10. Akhmediev, N., Eleonskii, V. M. \& Kulagin, N. E. Exact first-order solutions of the nonlinear Schrödinger equation. Theor. Math. Phys. 72, 809-818 (1987).

11. Dysthe, K. B. \& Trulsen, K. Note on breather type solutions of the NLS as models for freak-waves. Phys. Scripta 82, 48-52 (1999).

12. Karjanto, N. \& van Groesen, E. Mathematical physics properties of waves on finite background. Handbook of Solitons: Research, Technology and Applications: Lang, S.P. \& Bedore, S. H. Eds, Nova Science Publishers, Inc., New York, pp 509-539 (2009).

13. Zakharov, V. E., Dyachenko, A. I. \& Prokofiev, A. O. Freak waves as nonlinear stage of Stokes wave modulation instability. Eur. J. Mech. B - Fluids 5, 677-692 (2006).

14. Shrira, V. I. \& Geogjaev, Y. V. What make the Peregrine soliton so special as a prototype of freak waves?J. Eng. Math. 67, 11-22 (2010).

15. Osborne, A. R. Nonlinear ocean waves and the inverse scattering transform (Academic Press, 2010).

16. Dias, F., Bridges, T. J. \& Dudley, J. M. Rogue waves. Environmental Hazards: The Fluid Dynamics and Geophysics of Extreme Events, Moffatt, H. K. \& Shuckburgh, E. (Eds), Lecture Notes Series, Institute for Mathematical Sciences, National University of Singapore - Vol. 21 pp 295-306 (2011).

17. Solli, D. R., Ropers, C., Koonath, P. \& Jalali, B. Optical rogue waves. Nature 450 1054-1057 (2007)

18. Dudley, J. M., Genty, G., Dias, F., Kibler, B. \& Akhmediev, N. Modulation instability, Akhmediev Breathers and continuous wave supercontinuum generation. Opt. Express 17, 21497-21508 (2009).

19. Van Simaeys, G., Emplit, Ph. \& Haelterman, M. Experimental demonstration of the Fermi-Pasta-Ulam recurrence in a modulationally unstable optical wave. Phys. Rev. Lett. 87, 033902 (2001).

20. Fermi, E., Pasta, J. \& Ulam, S. Studies of the Nonlinear Problems, I, Los Alamos Report LA-1940, (1955), reprinted in Segre, E. Collected Papers of Enrico Fermi pp. 978-988 (University of Chicago Press, 1965).

21. Kibler, B., Fatome, J., Finot, C., Millot, G., Dias, F., Genty, G., Akhmediev, N. \& Dudley, J. M. The Peregrine soliton in nonlinear fibre optics. Nature Physics 6, 790-795 (2010).

22. Hammani, K., Kibler, B., Finot, C., Morin, P., Fatome, J., Dudley, J. M. \& Millot, G. Peregrine soliton generation and breakup in standard telecommunications fiber. Opt. Lett. 36, 112-114 (2011).

23. Lake, B. M., Yuen, H. C., Rungaldier, H. \& Ferguson, W. E. Nonlinear deep-water waves: theory and experiment. Part 2. Evolution of a continuous wave train. J. Fluid Mech. 83, 49-74 (1977).
24. Tulin, M. P. \& Waseda, T. Laboratory observations of wave group evolution, including breaking effects. J. Fluid Mech. 378, 197-232 (1999).

25. Karjanto, N. \& van Groesen, E. Qualitative comparisons of experimental results on deterministic freak wave generation based on modulational instability. J. Hydro-Environ. Res. 3,186-192 (2010).

26. Clauss, G. F., Klein, M. \& Onorato, M. Formation of extraordinarily high waves in space and time. Proceedings of the ASME 2011 30th International Conference on Ocean, Offshore and Arctic Engineering OMAE2011, June 19-24, 2011, Rotterdam, The Netherlands (2011).

27. Erkintalo, M., Genty, G., Wetzel, B. \& Dudley, J. M. Akhmediev breather evolution in optical fiber for realistic initial conditions. Phys. Lett. A 375, 2029-2034 (2011).

28. Grelu, Ph. \& Akhmediev, N. Dissipative solitons for mode-locked lasers. Nature Photon. 6, 84-92 (2012).

29. Dudley, J. M., Barry, L. P., Harvey, J. D., Thomson, M. D., Thomsen, B. C., Bollond, P. G. \& Leonhardt, R. Complete characterization of ultrashort pulse sources at $1550 \mathrm{~nm}$. IEEE J. Quant. Electron. 35, 441-450 (1999).

30. Han, Y. \& Jalali, B. Photonic time-stretched analog-to-digital converter fundamental concepts and practical considerations. IEEE J. Lightwave Technol. 21, 3085-3103 (2003).

31. Andrekson, P. A. \& Westlund, M. Nonlinear optical fiber based high resolution all-optical waveform sampling. Laser Photon. Rev. 1, 231-248 (2007).

\section{Acknowledgements}

We acknowledge support from the French Agence Nationale de la Recherche (ANR-2011-EMMA-005-01 SO FAST, ANR-09-BLAN-0065 IMFINI), the Academy of Finland Research grants 132279 and 130099, and the Australian Research Council Discovery Project scheme DP110102068. B. Kibler also thanks the support of the Conseil Régional de Bourgogne through the Photcom PARI grant. F.D. and J.M.D. acknowledge supprot from the European Research Council Project MULTIWAVE.

\section{Author contributions}

B. K., J. F., and C. F. performed experiments. The development of analytical tools and simulations was performed by B. K., G. M., G. G., F. D., N. A. and J. M. D. All authors participated in the analysis of the results and the writing of the paper.

\section{Additional information}

Competing financial interests: The authors declare no competing financial interests.

License: This work is licensed under a Creative Commons

Attribution-NonCommercial-NoDerivative Works 3.0 Unported License. To view a copy of this license, visit http://creativecommons.org/licenses/by-nc-nd/3.0/

How to cite this article: Kibler, B. et al. Observation of Kuznetsov-Ma soliton dynamics in optical fibre. Sci. Rep. 2, 463; DOI:10.1038/srep00463 (2012). 